\title{
Changes in Temporal and Spatial Patterns of Intrinsic Brain Activity and Functional Connectivity in Upper-Limb Amputees: An fMRI Study
}

\author{
Bingbo Bao ${ }^{1},{ }^{1}$ Lei Duan, ${ }^{2}$ Haifeng Wei, ${ }^{1}$ Pengbo Luo, ${ }^{1}$ Hongyi Zhu, ${ }^{1}$ Tao Gao, ${ }^{1}$ Xiaoer Wei, ${ }^{3}$ \\ Jing $\mathrm{Li}^{3}{ }^{3}$ Yuehua $\mathrm{Li}^{3}{ }^{3}$ Yimin Chai, ${ }^{1}$ Changqing Zhang, ${ }^{1}$ and Xianyou Zheng $\mathbb{D}^{1}$ \\ ${ }^{1}$ Department of Orthopedic Surgery, Shanghai Jiao Tong University Affiliated Sixth People's Hospital, Shanghai 200233, China \\ ${ }^{2}$ Department of Orthopedic Surgery, Yueyang Hospital, Shanghai University of Traditional Chinese Medicine, \\ Shanghai 200437, China \\ ${ }^{3}$ Institute of Diagnostic and Interventional Radiology, Shanghai Jiao Tong University Affiliated Sixth People's Hospital, \\ Shanghai 200233, China
}

Correspondence should be addressed to Xianyou Zheng; zhengxianyou@126.com

Received 30 August 2020; Revised 4 January 2021; Accepted 5 April 2021; Published 24 April 2021

Academic Editor: Carlo Cavaliere

Copyright (c) 2021 Bingbo Bao et al. This is an open access article distributed under the Creative Commons Attribution License, which permits unrestricted use, distribution, and reproduction in any medium, provided the original work is properly cited.

Background. Amputation in adults is a serious procedure or traumatic outcome, one that leads to a possible "remapping" of limb representations (somatotopy) in the motor and sensory cortex. The temporal and spatial extent underlying reorganization of somatotopy is unclear. The aim of this study was to better understand how local and global structural plasticity in sensorymotor cortical networks changes temporally and spatially after upper-limb amputation. Methods. We studied 8 healthy nonamputee control subjects and 16 complete upper-limb amputees. Resting-state MRI (rs-fMRI) was used to measure local and large-scale relative differences (compared to controls) in both the amplitude of low-frequency fluctuations (ALFF) and degree of centrality (DC) at 2 months, 6 months, and 12 months after traumatic amputation. Results. In amputees, rs-fMRI scans revealed differences in spatial patterns of ALFF and DC among brain regions over time. Significant relative increases in ALFF and DC were detected not only in the sensory and motor cortex but also in related cortical regions believed to be involved in cognition and motor planning. We observed changes in the magnitude of ALFFs in the pre- and postcentral gyrus and primary sensory cortex, as well as in the anterior cingulate, parahippocampal gyrus, and hippocampus, 2 months after the amputation. The regional distribution of increases/decreases in ALFFs and DC documented at 2-month postamputation was very different from those at 6 and 12-month postamputation. Conclusion. Local and wide-spread changes in ALFFs in the sensorimotor cortex and cognitive-related brain regions after upper-limb amputation may imply dysfunction not only in sensory and motor function but also in areas responsible for sensorimotor integration and motor planning. These results suggest that cortical reorganization after upper extremity deafferentation is temporally and spatially more complicated than previously appreciated, affecting DC in widespread regions.

\section{Introduction}

Not only is complete traumatic limb amputation in adults an emotionally disturbing wound, but it can also develop into a serious medical condition. The traumatic amputation event often causes widespread damage and destruction of skin, tendons, muscles, bones, vasculature, and nerves at the trauma site, and because nerves are axotomized, afferent neurons degenerate, terminal swelling ensues, and regenerative sprouting of the severed axons forms neuromas in the limb stump. This deafferentation also leads to structural and physiological changes in the spinal cord, and some previous studies suggest that a remapping, or reorganization, of limb representations (somatotopy) eventually occurs at the cortical level in motor 
and sensory networks. These changes are often accompanied by altered perceptual sensations reported by the patient.

Alterations in the sensorimotor cortex of limb amputees have been extensively investigated with various electrophysiological and neuroimaging techniques [1-5]. Almost $90 \%$ of amputees report "phantom sensations," which are described as a vague feeling that the missing upper or lower extremity is still present; some amputees experience severe pain in what seems to be the missing limb [6-9]. Transcranial magnetic stimulation combined with EEG recordings has demonstrated enhanced responses, or neural plasticity, in both motor and somatosensory cortexes in amputees with phantom limb pain [2]. Further, resting-state functional magnetic resonance imaging (rs-fMRI) studies have shown that phantom limb pain is correlated with primary sensorimotor functional remapping after amputation, suggesting that this functional reorganization represents an inappropriate adjustment to deafferentation.

Maladaptive plasticity in sensorimotor networks after nerve deafferentation $[10,11]$ is one hypothesis to explain the phenomenon of phantom pain. Reorganization of the somatotopic body map in the cortex seems to be an important element in distinguishing painful from nonpainful phantom sensations [10]. However, the widely accepted theory that maladaptive plasticity is the cause of phantom pain has been challenged recently. Rather than plasticity, unusually elevated neural activity in the affected sensorimotor regions of the amputated upper extremity may be responsible for phantom pain $[12,13]$. These results may indicate that a more complex and multifactorial cause may underlie phantom pain. Accordingly, studies have also investigated cortical plasticity in amputee patients without phantom pain $[5,14$, 15 ] and found that the alteration in the primary somatosensory cortex is not consistently related to pain symptoms [1].

In the last few decades, neuroimaging techniques like fMRI have been routinely used to detect and analyze functional and anatomical changes in the brain [16, 17], essentially probing the functional architecture of the brain. rs-fMRI is a type of fMRI that provides an estimate of the blood-oxygen-level dependent (BOLD) signal in the brain while subjects are awake, quiet, and not performing any task, and rs-fMRI can provide anatomical specificity of sensorimotor cortex subregional somatotopy $[18,19]$. The BOLD signals represent the hemodynamic response to neural activity [20]. The amplitude of low-frequency fluctuations (ALFFs) of the BOLD signal is thought to reflect the magnitude of intrinsic neural activity (local activity of individual regions or voxels) and can provide information about interregional functional connectivity. Thus, rs-fMRI research can reveal neurological processes that occur without external stimulation, and analysis of rs-fMRI signals can reveal information about resting-state connectivity of networks. Only a few studies have analyzed rs-fMRI connectivity and its other metrics such as ALFF to determine whole-brain changes after a traumatic event or in neurological pathology like Parkinson's disease.

In the present study, we used rs-fMRI to investigate local and global brain plasticity in patients with upper-limb amputations. Our aim in this study was to map out how local and global structural and functional connectivity in sensorymotor cortical networks and other brain regions change temporally and spatially after upper-limb amputation. We used two reliable and effective measures of rs-MRI: (1) the ALFF and (2) the degree of centrality (DC), the latter of which reflects the number of instantaneous functional connections between one region and the rest of the brain and thus how much a node influences entire brain areas. These parameters were used to indicate the intensity of spontaneous fluctuations in the BOLD signal at 2, 6, and 12 months after traumatic amputation.

\section{Methods}

2.1. Participants. Eight healthy limb-intact man and woman volunteers (control group) were recruited, along with 16 traumatic upper-limb amputees, between the ages of 24 years and 55 years (Table 1). The convenience sample of control subjects was recruited to be balanced by age and sex with the amputee group. A detailed medical history, together with careful examination of head CT scans, confirmed that neither amputees nor controls showed evidence of brain lesions, and their medical history showed no evidence of neurological or psychiatric illness.

All procedures used in this study were approved by the Committee for Medical Ethics of Shanghai Jiao Tong University, Affiliated Sixth People's Hospital, and followed the Ethical Principles for Medical Research Involving Human Subjects (WMA-Declaration of Helsinki). Written informed consent was obtained from all study participants.

2.2. Data Acquisition and Scanning Procedures. Scans were acquired on a SIEMENS TRIO 3-Tesla scanner in the Shanghai Sixth People's Hospital, Shanghai Jiao Tong University (SJTU). Anatomical data was acquired using a T1-weighted magnetization prepared rapid acquisition gradient echo sequence with the following parameters: $\mathrm{TR}=1900 \mathrm{~ms}, \mathrm{TE}=2.52 \mathrm{~ms}$, flip angle $=9^{\circ}$, and voxel size $=1 \mathrm{~m}$ misotropic resolution. Functional data based on the BOLD signal were acquired using a multiple gradient echo planar $\mathrm{T} 2 *$-weighted pulse sequence, with the following parameters: $\mathrm{TR}=3000 \mathrm{~ms}, \mathrm{TE}=21 \mathrm{~ms}$, flip angle $=90^{\circ}$, imaging matrix $=96 \times 96$, and FOV $=210$ mm axial slices. 40 slices with slice thickness of $2.7 \mathrm{~mm}$ and no gap were oriented in the oblique axial plane, covering the whole cortex, with partial coverage of the cerebellum. A total of 240 volumes were collected, during which participants were asked to lie still in a dimmed room with their eyes open. They were explicitly asked not to move any body parts.

2.3. $r s-f M R I$ Data Preprocessing. rs-fMRI data of each subject were preprocessed with the software Data Processing Assistant for Resting-State fMRI (DPARSF) [21], which is based on Statistical Parametric Mapping software, SPM8 (Wellcome Trust Centre for Neuroimaging, London, UK; http:// www.fil.ion.ucl.ac.uk/spm) and the rs-fMRI Data Analysis Toolkit [22]. Functional image preprocessing was executed according to the following steps: (1) the first 10 volumes were discarded from analysis, since the subjects were adapting to the noise of the scanner and the test situation; this 
TABle 1: Demographic and clinical characteristics of patients with upper-limb amputation $(n=16)$.

\begin{tabular}{|c|c|c|c|c|c|c|c|c|c|}
\hline \multirow{2}{*}{$\begin{array}{l}\text { No. } \\
1\end{array}$} & \multirow{2}{*}{$\begin{array}{l}\text { Sex } \\
M\end{array}$} & \multirow{2}{*}{$\frac{\text { Age }(\mathrm{yr})}{35}$} & \multicolumn{2}{|c|}{$\begin{array}{l}\text { Amputated side } \\
\text { (dominant side) }\end{array}$} & \multicolumn{2}{|c|}{$\begin{array}{l}\text { Amputation level } \\
\text { (\% limb } \\
\text { remaining) }\end{array}$} & \multicolumn{3}{|c|}{$\begin{array}{l}\text { Elapsed time after } \\
\text { amputation (mo.) for } \\
\text { rs-fMRI scans }\end{array}$} \\
\hline & & & $R$ & (R) & $\mathrm{P}$ & (16) & 2 & 6 & 12 \\
\hline 2 & M & 33 & $\mathrm{~L}$ & (R) & $\mathrm{P}$ & (25) & 2 & 6 & 12 \\
\hline 3 & M & 49 & $\mathrm{~L}$ & (R) & $\mathrm{P}$ & (20) & 2 & 6 & 12 \\
\hline 4 & $\mathrm{~F}$ & 45 & $\mathrm{R}$ & (R) & $\mathrm{P}$ & (30) & 2 & 6 & 12 \\
\hline 5 & F & 47 & $\mathrm{R}$ & (R) & D & (45) & 2 & 6 & 12 \\
\hline 6 & $\mathrm{~F}$ & 46 & $\mathrm{R}$ & (R) & D & (72) & 2 & 6 & 12 \\
\hline 7 & M & 24 & $\mathrm{R}$ & (R) & $\mathrm{P}$ & (28) & 2 & 6 & 12 \\
\hline 8 & M & 40 & $\mathrm{R}$ & (R) & D & (66) & 2 & 6 & 12 \\
\hline 9 & $\mathrm{M}$ & 29 & $\mathrm{R}$ & (R) & D & (58) & 2 & 6 & 12 \\
\hline 10 & $\mathrm{~F}$ & 55 & $\mathrm{~L}$ & (R) & D & (50) & 2 & 6 & 12 \\
\hline 11 & F & 49 & $\mathrm{R}$ & (R) & $\mathrm{P}$ & (18) & 2 & 6 & 12 \\
\hline 12 & M & 48 & $\mathrm{~L}$ & (R) & D & (65) & 2 & 6 & 12 \\
\hline 13 & $\mathrm{M}$ & 35 & $\mathrm{R}$ & (R) & D & (44) & 2 & 6 & 12 \\
\hline 14 & M & 30 & $\mathrm{~L}$ & (R) & D & (40) & 2 & 6 & 12 \\
\hline 15 & M & 50 & $\mathrm{~L}$ & (R) & D & (34) & 2 & 6 & 12 \\
\hline 16 & M & 44 & $\mathrm{~L}$ & (R) & $\mathrm{P}$ & (12) & 2 & 6 & 12 \\
\hline
\end{tabular}

M: male; F: female; L: left; R: right; D: distal; P: proximal.

TABLE 2: Relative regional changes in ALFFs at 2 months* $^{*}$ in upper-limb amputees $(n=16)$ compared to normal control subjects $(n=8)$.

\begin{tabular}{ccccccc}
\hline \multirow{2}{*}{ Direction of change } & Brain region & \multirow{2}{*}{ Voxels* } & $t$-value & \multicolumn{2}{c}{ MNI coordinates } \\
& Hippocampus_L & 189 & 4.257 & -21 & -27 & -12 \\
& ParaHippocampal_L & 189 & 3.452 & -21 & -9 & -30 \\
& Cingulate_Ant_R & 100 & 3.295 & 3 & 27 & 0 \\
Positive ALFF & ParaHippocampal_R & 49 & 4.258 & 18 & 0 & -21 \\
& Putamen_L & 34 & 3.489 & -27 & -15 & 3 \\
& Insula_R & 19 & 3.583 & 42 & -9 & -9 \\
& Putamen_R & 16 & 3.334 & 27 & 3 & -3 \\
& Postcentral_L & 11 & 3.414 & -63 & -21 & 18 \\
& Supp_Motor_Area_L & 8 & 3.729 & -3 & 21 & 54 \\
& Precentral_R & 97 & -4.332 & 42 & -12 & 57 \\
Negative ALFF & Precuneus_R & 51 & -4.492 & 6 & -66 & 54 \\
& Precentral_L & 51 & -4.128 & -27 & 0 & 60 \\
& Postcentral_R & 44 & -3.836 & 27 & -42 & 63 \\
& Sup_Motor_Area_R & 42 & -3.705 & 3 & -15 & 72 \\
& Postcentral_L & 19 & -3.758 & -21 & -45 & 69 \\
\hline
\end{tabular}

${ }^{*}$ rs-fMRI scan done 2-month postamputation; MNI: Montreal Neurological Institute; ${ }^{*}$ number of voxels exceeding threshold (cluster size).

preacquisition period also allowed the scanner to stabilize. The number of time points for this period was not less than 230; (2) slice scan time corrections were made; (3) head movement corrections were made; (4) spatial normalization was done, in which the corrected images were then registered to the T1 structural image, and spatially normalized into the standard Montreal Neurological Institute template (resampled into $3 \times 3 \times 3 \mathrm{~mm}^{3}$ ); (5) nuisance variables, including white matter signals and head motions, were measured using the
Friston-24 model, and cerebrospinal fluid signals were removed by regression; (6) spatial smoothing using a $6 \mathrm{~mm}$ FWHM Gaussian kernel was performed; (7) finally, linear trends in the time series were removed.

2.4. ALFF Calculations. To study the relationship between functional plasticity after upper extremity deafferentation and resting-state brain functions, we employed ALFF analysis to explore which resting-state networks might be 
TABLE 3: Relative regional changes in ALFFs at 6 months*.

\begin{tabular}{|c|c|c|c|c|c|c|}
\hline \multirow{2}{*}{ Direction of change } & \multirow{2}{*}{ Brain region } & \multirow{2}{*}{ Voxels } & \multirow{2}{*}{$t$-value } & \multicolumn{3}{|c|}{ MNI coordinates } \\
\hline & & & & $x$ & $y$ & $z$ \\
\hline \multirow{5}{*}{ Positive ALFF } & Putamen_L & 88 & 4.993 & -21 & -3 & 3 \\
\hline & Hippocampus_L & 36 & 3.804 & -30 & -21 & -21 \\
\hline & Caudate_R & 17 & 3.924 & 15 & 15 & 3 \\
\hline & Putamen_R & 11 & 3.386 & 24 & 6 & -6 \\
\hline & Supp_Motor_Area_L & 10 & 3.521 & -3 & 24 & 54 \\
\hline \multirow{8}{*}{ Negative ALFF } & Paracentral_Lobule_L & 903 & -6.797 & -15 & -36 & 63 \\
\hline & Supp_Motor_Area_R & 903 & -5.979 & 6 & -6 & 60 \\
\hline & Lingual_R & 428 & -5.184 & 6 & -72 & -9 \\
\hline & Cuneus_R & 428 & -4.449 & 9 & -81 & 24 \\
\hline & Precuneus_R & 86 & -5.02 & 15 & -66 & 39 \\
\hline & Fusiform_R & 47 & -3.948 & 24 & 9 & -45 \\
\hline & Precentral_R & 30 & -5.233 & 48 & -3 & 42 \\
\hline & Temporal_Inf_R & 27 & -3.21 & 42 & -57 & -9 \\
\hline
\end{tabular}

*rs-fMRI scan done 6-month postamputation.

TABLE 4: Relative regional changes in ALFFs at 12 months.

\begin{tabular}{|c|c|c|c|c|c|c|}
\hline \multirow{2}{*}{ Direction of change } & \multirow{2}{*}{ Brain region } & \multirow{2}{*}{ Voxels } & \multirow{2}{*}{$t$-value } & \multicolumn{3}{|c|}{ MNI coordinates } \\
\hline & & & & $x$ & $y$ & $z$ \\
\hline \multirow{16}{*}{ Positive ALFF } & Caudate_R & 202 & 3.91 & 15 & 12 & 3 \\
\hline & Rectus_L & 202 & 3.835 & 3 & 30 & -18 \\
\hline & Cingulate_Mid_L & 95 & 4.198 & -12 & -42 & 42 \\
\hline & Cingulate_Ant_R & 69 & 3.1 & 3 & 18 & 21 \\
\hline & Frontal_Sup_Medial_R & 41 & 3.936 & 6 & 57 & 27 \\
\hline & ParaHippocampal_L & 32 & 3.362 & -27 & -39 & -12 \\
\hline & Cingulate_Mid_R & 30 & 6.424 & 12 & -33 & 42 \\
\hline & Thalamus_R & 30 & 3.805 & 15 & -9 & -3 \\
\hline & Precuneus_L & 28 & 4.674 & -9 & -69 & 60 \\
\hline & Pallidum_L & 25 & 4.531 & -21 & -3 & 0 \\
\hline & Occipital_Mid_R & 24 & 3.614 & 51 & -66 & 24 \\
\hline & Caudate_R & 18 & 5.593 & 21 & -3 & 18 \\
\hline & Supp_Motor_Area_L & 16 & 4.509 & -3 & 21 & 54 \\
\hline & Hippocampus_R & 14 & 4.155 & 36 & -30 & -6 \\
\hline & Putamen_L & 12 & 3.368 & -27 & -15 & 3 \\
\hline & Insula_R & 10 & 3.873 & 39 & -18 & 0 \\
\hline \multirow{9}{*}{ Negative ALFF } & Lingual_R & 476 & -6.267 & 18 & -63 & -6 \\
\hline & Fusiform_R & 102 & -5.381 & 24 & 3 & -45 \\
\hline & Lingual_L & 67 & -3.192 & -12 & -75 & -6 \\
\hline & Precuneus_R & 55 & -4.646 & 24 & -45 & 6 \\
\hline & Postcentral_R & 41 & -6.032 & 27 & -42 & 63 \\
\hline & Precentral_R & 29 & -6.454 & 30 & -21 & 63 \\
\hline & Precentral_L & 26 & -4.588 & -21 & -18 & 63 \\
\hline & Supp_Motor_Area_R & 25 & -5.477 & 3 & -9 & 57 \\
\hline & Paracentral_Lobule_L & 13 & -3.299 & -18 & -30 & 66 \\
\hline
\end{tabular}

${ }^{*}$ rs-fMRI scan done 12-month postamputation. 


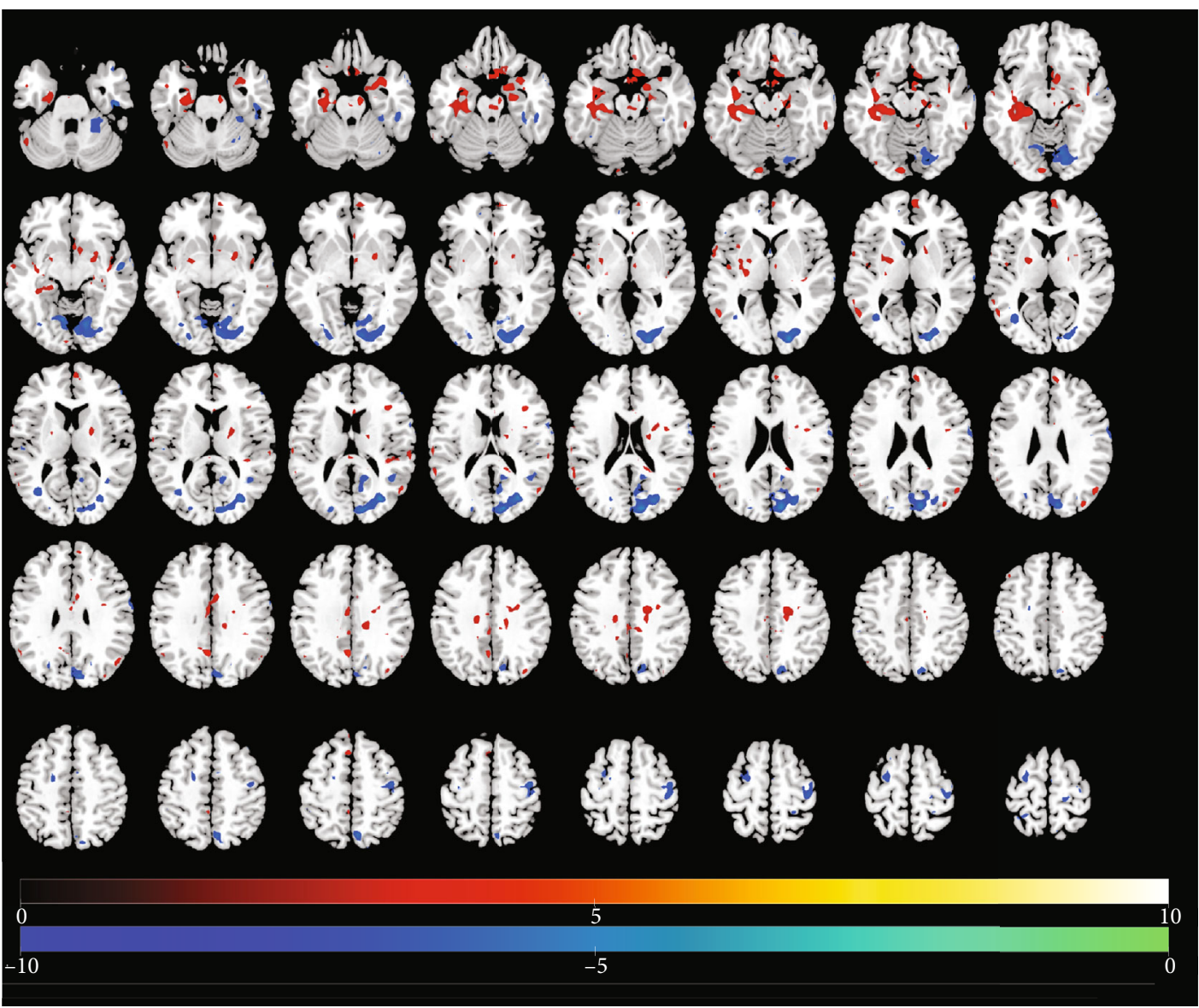

FIgURE 1: Identification of changed regional brain activity 2 months after traumatic amputation by using rs-fMRI. Relative magnitude of ALFF between upper-limb amputee patients $(n=16)$ and normal control subjects $(n=8)$ is plotted in axial images (top to bottom, inferior to superior). Colors toward red indicate increases in ALFF (significant $t$-values), and colors toward blue indicate significant decreases in ALFF relative to controls (cf. Table 2).

differentially activated in amputees compared to healthy control subjects. ALFF is calculated from the BOLD time course at each voxel [23]. The ALFF calculation was performed identically to that described in the studies of Zang et al. [24, 25]. ALFF was calculated for the traditional low-frequency band $(0.01-0.08 \mathrm{~Hz})$.

2.5. DC Calculations. DC reflects the strength of connections for a given voxel with all other voxels in the brain and can thus capture its relationship with the whole brain network at the voxel level; it represents a node characteristic of large-scale brain intrinsic connectivity networks [26]. Compared to a binary version of DC, weighted DC provides a more precise centrality characterization of functional brain networks [27]. Therefore, we used weighted DC. Pearson's correlations were calculated between the time courses of each voxel with that of every other voxel in the entire brain. Correlation coefficients with $r>0.2$ were summed for each voxel, and then, a weighted DC was obtained for each voxel. The threshold of 0.2 was used to eliminate counting voxels that had low temporal correlation. It has been shown that different threshold selections do not qualitatively change the results [28]. Spatial smoothing (FWHM $=6 \mathrm{~mm})$ was carried out after DC calculations, since lack of spatial smoothing may lead to artificial local correlations. We used the following formula for this DC calculation:

$$
D=\sum a i j, \quad j=1, \cdots, N, i \neq j, a i j=\left\{\begin{array}{lc}
0, & a i j<0.2 \\
a i j, & a i j \geq 0.2
\end{array}\right.
$$

In line with previous rs-fMRI studies, we removed negative correlations [29]. Since the physiological basis of negative correlations is uncertain, it was not calculated [30-32].

2.6. Statistical Analysis. Two-sample $t$-tests were used on the two indices of the BOLD signal (ALFF and DC) separately. The results were corrected for multiple comparisons with a combined threshold of a single voxel $(P<0.05)$ with Gaussian random field (GRF) correction.

\section{Results}

3.1. Demographic and Clinical Characteristics of Subjects. Table 1 shows basic demographic and clinical characteristics 


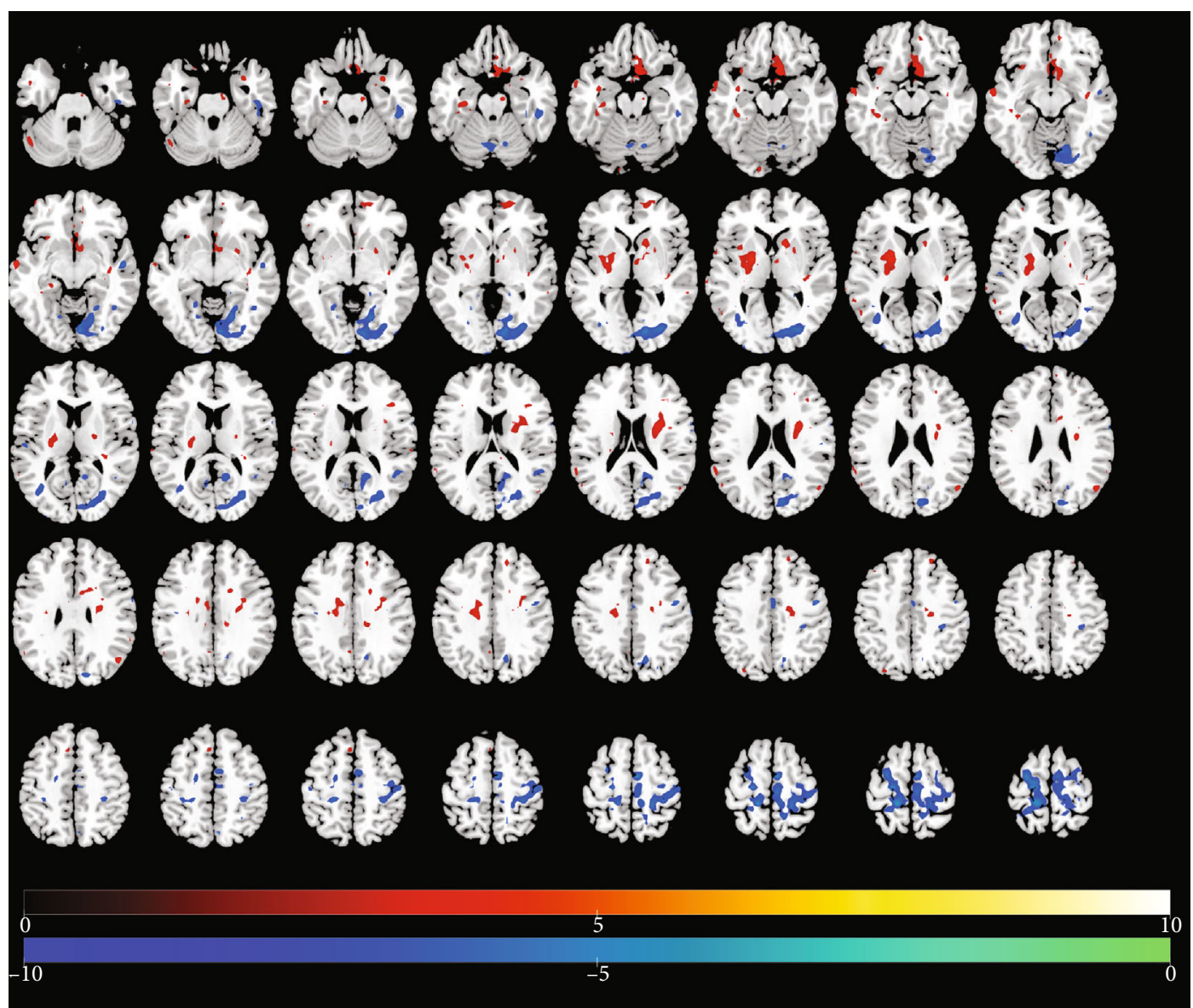

FIGURE 2: Changes in regional brain activity 6 months after traumatic amputation by using rs-fMRI. Differences in the magnitude of ALFF between the same upper-limb amputee patients and control subjects. Same conventions as in Figure 1.

of the amputees contributing data for this study. The mean age of the 8 healthy control subjects and 16 upper-limb amputees was statistically indistinguishable (37.9 years vs. 41.2 years, $P=0.07>0.05$ ). Also, the two groups were reasonably well balanced by sex, with 3 females and 5 males in the control group and 5 females and 11 males in the amputee group.

3.2. Changes in ALFF Over Time. Tables 2-4 present results of significant regional brain changes in ALFFs at 2-, 6-, and 12-month postamputation, respectively. Figures $1-3$ are the corresponding axial images of rs-fMRIs showing relative changes in ALFFs of amputees compared to control subjects.

3.2.1. Two-Month Postamputation. In rs-fMRIs, we observed the largest positive ALFFs in the temporal lobe, cingulate gyrus, pre- and post-central gyri, and precuneus (Table 2, Figure 1). Compared to normal controls at 2-month posttraumatic amputation, in upper-limb amputee patients, we detected significant positive ALFFs in the right side of the brain in the anterior cingulate, parahippocampal gyrus, insula, and putamen, while in the left side at this time point, significant positive ALFFs were detected in the hippocampus, parahippocampal gyrus, putamen, postcentral gyrus, and supplementary motor area. Significant negative ALFFs at 2 months were detected in the right side of the brain in the precentral gyrus, precuneus, and supplementary motor area, while in the left side at this time point, significant negative ALFFs were detected in the precentral gyrus and postcentral gyrus.

3.2.2. Six-Month Postamputation. Six months after the first rs-fMRI scans, a different pattern of regional ALFF changes emerged. In general, the large positive ALFFs we observed in the temporal lobe regions at 2 months were no longer detectable at 6 months or were much reduced in voxel extent (e.g., left hippocampus). One exception was a large relative increase in a negative ALFF in the right supplementary motor area from 42 voxels at 2 months to 903 voxels at 6 months (Table 3, Figure 2). At the 6-month time point, the largest changes were significant negative ALFFs in the paracentral lobule, supplementary motor area, lingual area, and cuneus. Other notable significant positive changes in ALFFs were in the left putamen, right caudate, and negative ALFFs in the fusiform gyrus and inferior temporal lobe.

3.2.3. Twelve-Month Postamputation. In general, at 12month postamputation, more significant positive and negative changes in ALFFs were detected, but the voxel extents tended to be smaller, and some previously undetected regions 


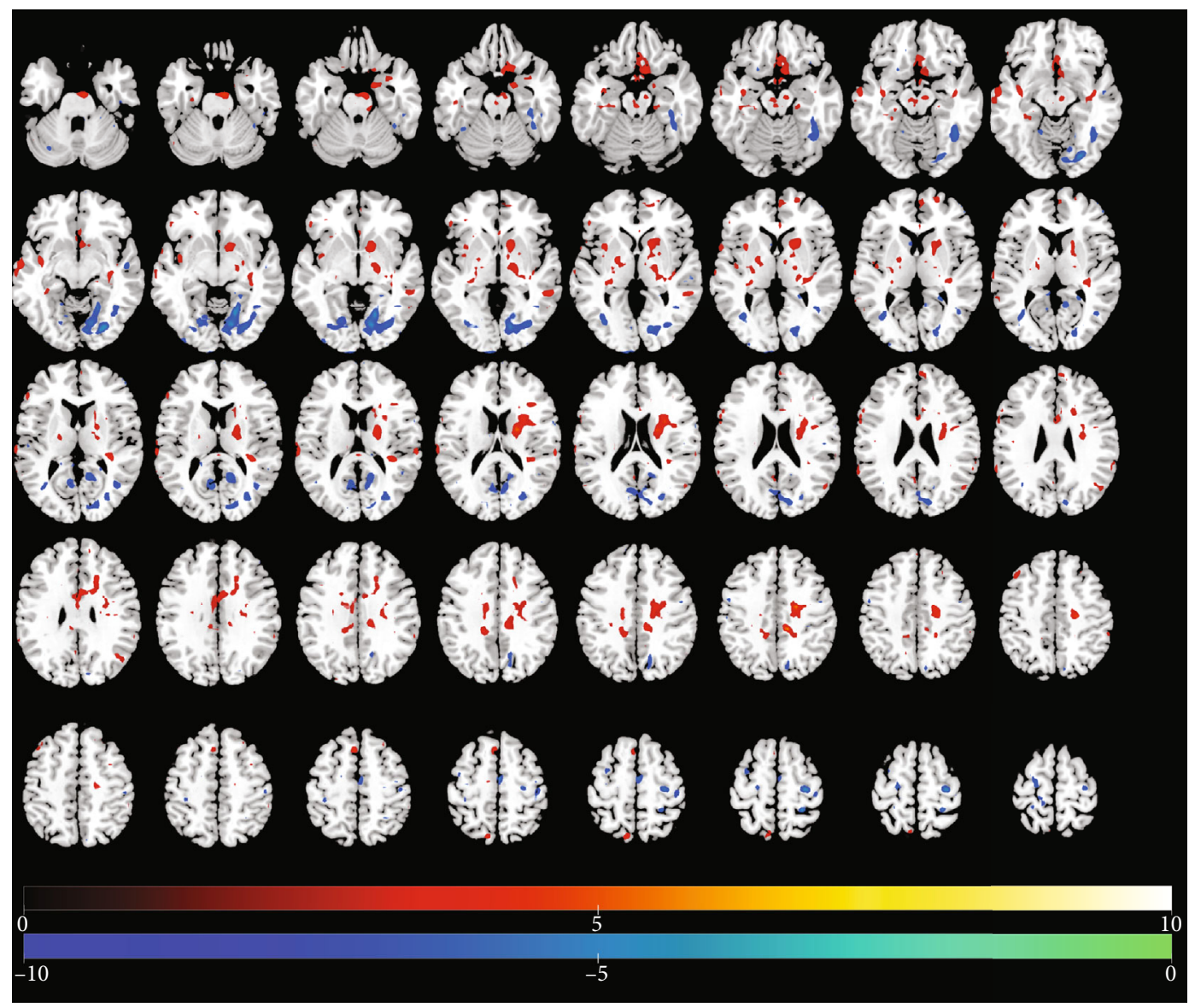

FIGURE 3: Changes in regional brain activity 12 months after traumatic amputation by using rs-fMRI. Differences in the magnitude of ALFF between the same upper-limb amputee patients and control subjects. Same conventions as in Figure 1.

TABLE 5: Relative regional changes in DC at 2 months.

\begin{tabular}{|c|c|c|c|c|c|c|}
\hline \multirow{2}{*}{ Direction of change } & \multirow{2}{*}{ Brain region } & \multirow{2}{*}{ Voxels } & \multirow{2}{*}{$t$-value } & \multicolumn{3}{|c|}{ MNI coordinates } \\
\hline & & & & $x$ & $y$ & $z$ \\
\hline \multirow{4}{*}{ Positive DC } & Cingulate_Mid_L & 27 & 3.373 & -6 & 12 & 39 \\
\hline & Frontal_Med_Orb_R & 12 & 3.29 & 9 & 42 & -12 \\
\hline & Rolandic_Oper_L & 11 & 4.459 & -63 & -3 & 12 \\
\hline & Postcentral_L & 10 & 3.603 & -63 & -21 & 27 \\
\hline \multirow{7}{*}{ Negative DC } & Precuneus_R & 105 & -4.736 & 15 & -48 & 21 \\
\hline & ParaHippocampal_R & 105 & -3.788 & 18 & -39 & -6 \\
\hline & Caudate_R & 23 & -4.711 & 21 & 24 & -6 \\
\hline & Precuneus_L & 23 & -3.808 & -9 & -60 & 15 \\
\hline & Occipital_Mid_L & 18 & -4.159 & -27 & -78 & 0 \\
\hline & Frontal_Inf_Tri_R & 10 & -3.876 & 51 & 24 & 6 \\
\hline & Precentral_R & 10 & -3.426 & 60 & 9 & 15 \\
\hline
\end{tabular}

emerged at this 12-month scan (Table 4, Figure 3). Exceptions are large positive ALFF changes in the right caudate and left rectus gyrus of the frontal lobe, and large negative ALFF changes in the right lingual area and fusiform gyrus. Interestingly, while the function of the rectus gyrus is unclear, it may be involved in higher cognitive function. We observed the largest positive ALFFs in the cingulate gyrus, pre- and post-central gyri, and precuneus. Persistent significant relative changes in ALFFs were detected in the sensorimotor cortex and some temporal lobe regions (Table 4, Figure 3) across the 2-, 6-, and 12-month scans.

3.3. Changes in DC Over Time. Tables 4-6 present results of significant regional brain changes in DCs at 2-, 6-, and 12- 
TABLE 6: Relative regional changes in DC at 6 months.

\begin{tabular}{|c|c|c|c|c|c|c|}
\hline \multirow{2}{*}{ Direction of change } & \multirow{2}{*}{ Brain region } & \multirow{2}{*}{ Voxels } & \multirow{2}{*}{$t$-value } & \multicolumn{3}{|c|}{ MNI coordinates } \\
\hline & & & & $x$ & $y$ & $z$ \\
\hline \multirow{5}{*}{ Positive DC } & Calcarine_R & 103 & 5.182 & 18 & -99 & 0 \\
\hline & Cuneus_L & 19 & 6.229 & 0 & -90 & 33 \\
\hline & ParaHippocampal_L & 14 & 3.29 & -18 & 3 & -33 \\
\hline & Postcentral_L & 9 & 4.074 & -63 & -21 & 24 \\
\hline & Postcentral_R & 6 & 3.138 & 45 & -42 & 63 \\
\hline \multirow{8}{*}{ Negative DC } & Precuneus_R & 31 & -4.937 & 21 & -51 & 18 \\
\hline & ParaHippocampal_R & 27 & -4.193 & 21 & -39 & -6 \\
\hline & Hippocampus_R & 15 & -4.685 & 27 & -21 & -15 \\
\hline & Precuneus_L & 15 & -4.31 & -3 & -54 & 69 \\
\hline & Cingulate_Mid_R & 14 & -5.296 & 12 & -6 & 39 \\
\hline & Cingulate_Mid_L & 11 & -3.602 & -12 & -33 & 42 \\
\hline & Postcentral_L & 6 & -3.025 & -30 & -33 & 54 \\
\hline & Caudate_R & 5 & -4.04 & 21 & 24 & -6 \\
\hline
\end{tabular}

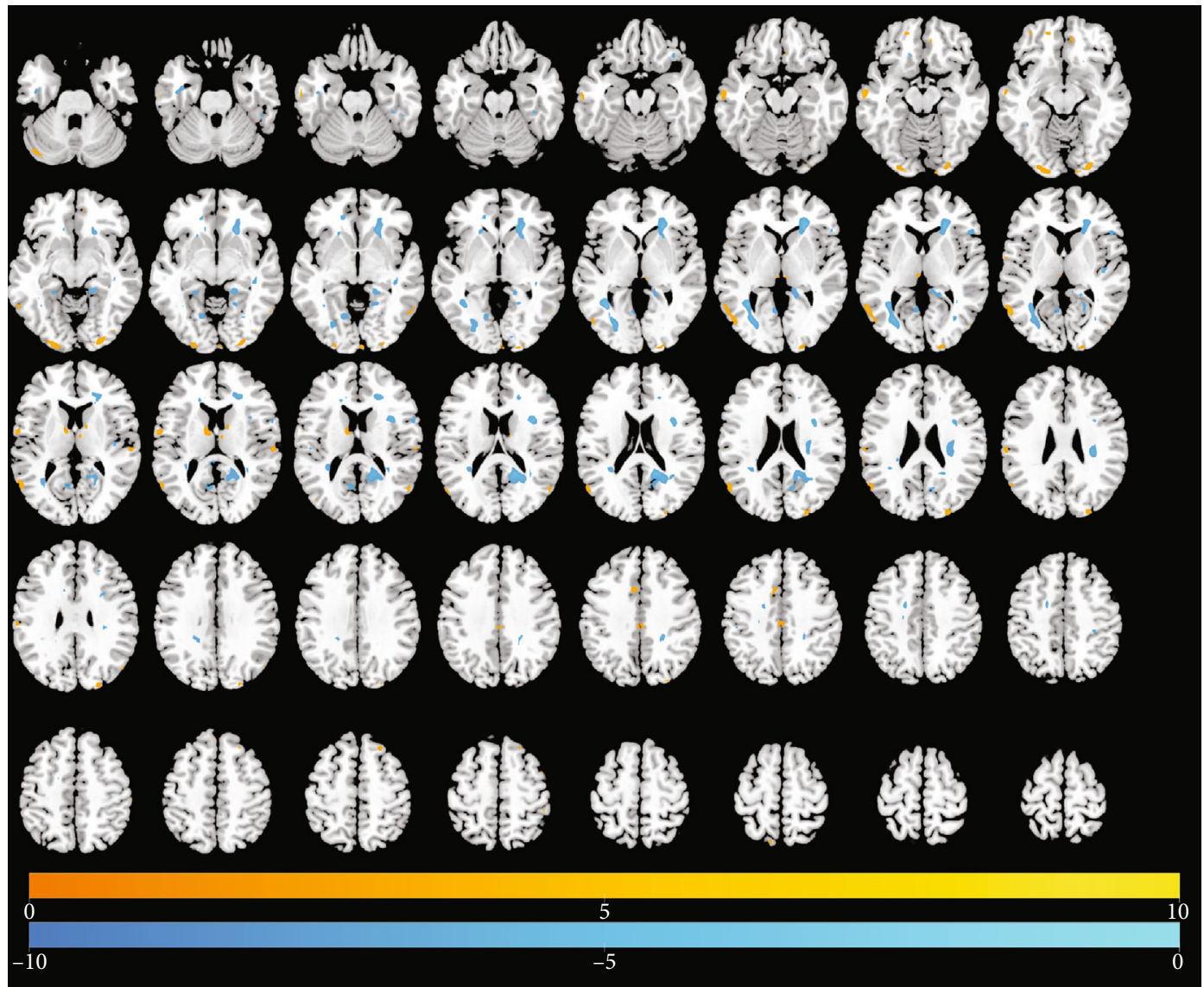

FIgURE 4: Differences in the magnitude of DC between the same upper-limb amputee patients and control subjects at 2 months of rs-fMRI scan. Same conventions as in Figure 1.

month postamputation, respectively. Figures 4-6 are the corresponding axial images of rs-fMRIs showing relative changes in DCs of amputees compared to control subjects.

3.3.1. Two-Month Postamputation. In rs-fMRIs, we observed the largest positive changes in DC in the left midcingulate gyrus and the largest negative changes in the right precuneus and right parahippocampal gyri (Table 5, Figure 4). In general, the spatial extent of DC changes at 2-month postamputation was much smaller than the corresponding ALFFs at 2 months (cf. Figures 1 and 4). Smaller significant positive or negative changes in DCs were detected in the rolandic 


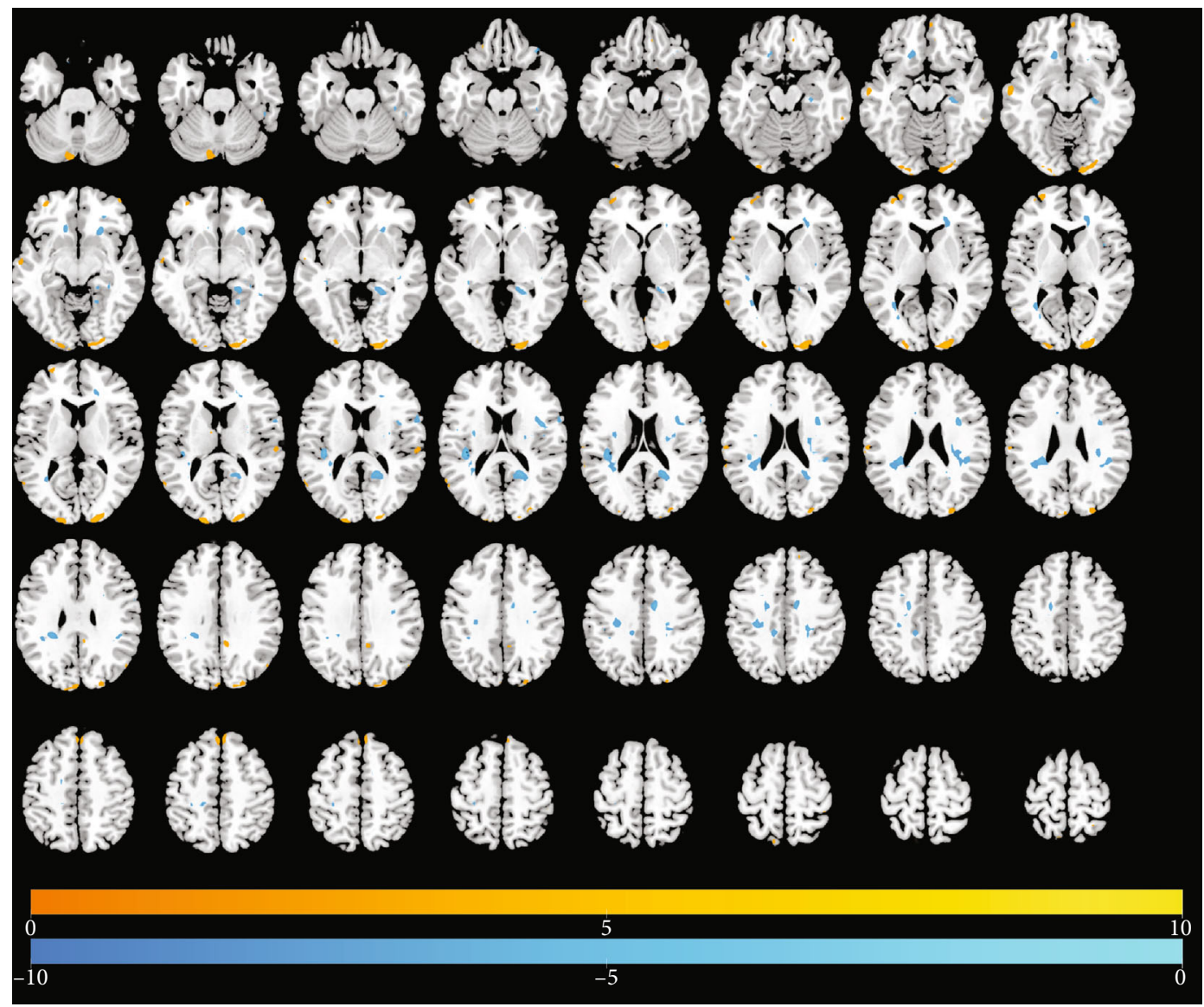

Figure 5: Differences in the magnitude of DC between the same upper-limb amputee patients and control subjects at 6 months of rs-fMRI scan. Same conventions as in Figure 1.

operculum, frontal medial orbital cortex, precuneus, parahippocampal gyrus, caudate, midoccipital cortex, and right parts of the triangularis of the inferior frontal gyrus.

3.3.2. Six-Month Postamputation. Six months after the first rs-fMRI scans, a different pattern of regional functional connectivity emerged. At this time point, we detected a new large positive DC in the right calcarine, and other positive and negative DCs were on the same order of magnitude as those observed at 2 months (Table 6, Figure 5).

3.3.3. Twelve-Month Postamputation. At the final scan at 12 months, we detected large positive changes in DC in the right lingual and cuneus and large negative changes in the parahippocampal gyrus and precuneus (Table 7, Figure 6). These changes in functional connectivity either were absent in earlier scans or were much smaller in magnitude.

\section{Discussion}

The temporal and spatial extents of cortical somatotopic reorganization after upper-limb amputation are unclear. In the present study, we described changes in spatial patterns of intrinsic brain activity (ALFFs) and functional connectivity (DC) by measuring rs-fMRI in traumatic upper-limb amputees at 2 months, 6 months, and 12 months. Relative to ALFFs and DC in normal controls, selective increases occurred in the sensory and motor cortex, as expected, but also increases and decreases occurred in related brain regions involved in functional plasticity after upper extremity deafferentation. The regional distribution of increases/decreases in ALFFs and DC documented at 2-month postamputation was very different from those at 6 and 12 months.

The sensory and motor brain networks of the human brain are somatotopically organized. A drastic upper extremity injury in humans, like traumatic amputation, may change the somatotopy of the primary motor cortex and primary sensory cortex of the deafferented hemisphere. Denervation due to amputation or nerve injury disrupts normal sensorimotor function. It is reported that cortical reorganization in the sensorimotor cortex, where cortical maps of intact body parts "expand" into areas associated previously with afferents of the missing limb, also has been reported in numerous animal studies of experimental amputation [33-35].

Sensorimotor reorganization is also observed in some transcranial magnetic stimulation studies in amputees. In these studies, increased excitability in motor areas contralateral to the amputated limb occurs. Remaining stump muscles have higher response amplitudes that can be evoked by transcranial magnetic stimulation applied in a larger area on the scalp than those responses in the intact arm $[36,37]$. In addition, a shift in the somatotopy of the lip [38], chin [39], and 


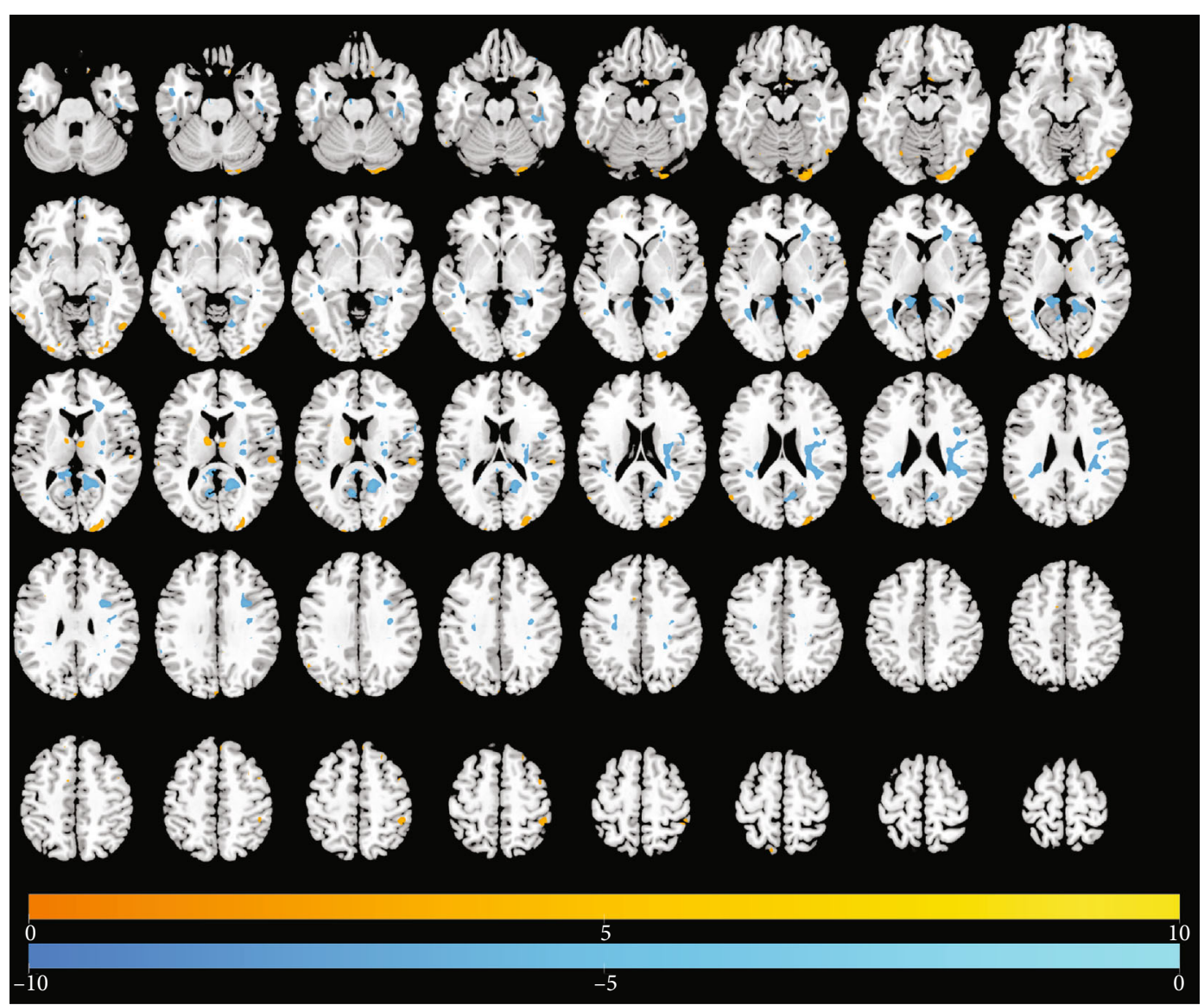

FIGURE 6: Differences in the magnitude of DC between the same upper-limb amputee patients and control subjects at 12 months of rs-fMRI scan. Same conventions as in Figure 1.

TABLE 7: Relative regional changes in DC at 12 months.

\begin{tabular}{|c|c|c|c|c|c|c|}
\hline \multirow{2}{*}{ Direction of change } & \multirow{2}{*}{ Brain region } & \multirow{2}{*}{ Voxels } & \multirow{2}{*}{$t$-value } & \multicolumn{3}{|c|}{ MNI coordinates } \\
\hline & & & & $x$ & $y$ & $z$ \\
\hline \multirow{6}{*}{ Positive DC } & Lingual_R & 188 & 6.163 & 24 & -93 & -15 \\
\hline & Cuneus_R & 188 & 5.813 & 18 & -99 & 9 \\
\hline & Fusiform_L & 23 & 4.308 & -21 & 3 & -45 \\
\hline & Parietal_Inf_R & 21 & 3.916 & 45 & -39 & 54 \\
\hline & Caudate_L & 12 & 4.725 & -12 & -3 & 15 \\
\hline & Postcentral_L & 10 & 4.03 & -21 & -30 & 78 \\
\hline \multirow{8}{*}{ Negative DC } & ParaHippocampal_R & 162 & -8.29 & 21 & -39 & -6 \\
\hline & Precuneus_R & 162 & -4.969 & 12 & -63 & 24 \\
\hline & Cingulate_Post_L & 56 & -6.593 & -6 & -39 & 12 \\
\hline & Fusiform_R & 47 & -4.827 & 39 & -33 & -18 \\
\hline & Precuneus_L & 25 & -5.595 & -6 & -60 & 15 \\
\hline & Frontal_Inf_Oper_R & 20 & -4.427 & 36 & 6 & 27 \\
\hline & Frontal_Inf_Tri_R & 16 & -5.115 & 51 & 24 & 6 \\
\hline & Putamen_R & 12 & -3.446 & 30 & -6 & 6 \\
\hline
\end{tabular}

shoulder [40] representation into the deafferented cortical hand area of upper-limb amputees has been reported using magnetoencephalography (MEG) and fMRI.
Denervation does not lead to a complete loss of the affected limb cortical representation, since the sensorimotor cortex still seems to be working with so-called "attempted 
movement" by the amputee. When an amputee tries to "move" their phantom limb, activation in the corresponding sensorimotor areas appears in fMRI, and this activation is similar to real executed movements in able-bodied subjects [38, 41, 42].

It has been shown that the persistent cortical representation of upper-limb amputees is relatively detailed for the postcentral gyrus and parietal cortex. For example, in a tetraplegic patient, movement trajectories and movement goals have been successfully decoded from electrophysiological activity in the posterior parietal cortex using intracranial recordings [43]. A persistent hand representation in S1 was also reported in a long-term spinal cord injury patient as measured by microstimulation [44]. Also, the topography of a persistent individual finger representation of a phantom hand has been shown in the somatosensory cortex of amputees [45].

ALFF measures the amplitude of time series fluctuations at each voxel, and DC represents the large-scale intrinsic connectivity of the brain at the voxel level. These measures of rsfMRI probe brain activity from different perspectives. In our investigation, we found that the ALFF and DC overlap spatially, which mainly occurred in the left superior frontal gyrus (Frontal_Sup_L). This result may suggest that changes in cognition may occur in amputation patient, and may have an extended impact on the brain plasticity.

We also found that the ALFFs in the primary motor cortex and primary sensory cortex decreased at 2, 6, and 12 months after the amputation. The brain regions involved with sensorimotor integration increased, specifically in the putamen, caudate, and precuneus. We speculate that this could represent a compensatory pattern after amputation. The deactivation of sensorimotor cortex may influence the associate cortex of sensory feedback. Since DC values decreased in the precuneus, caudate, and postcentral gyrus, this may indicate that sensorimotor integration is also impaired after limb amputation. It is expected that the primary motor cortex and the primary sensory cortex were silent after the deafferentation of the injured limb. Afterwards, the connection in the sensorimotor network at the affected hemisphere was diminished. In general, we found that amputation patients had significant brain function alterations in local regions and extensive alterations in widespread brain regions, such as corpus striatum and cognitionemotion cortex.

This study had several limitations. First, the sample size of the normal controls and amputation patients was relatively small, which may reduce confidence in the results. Therefore, more subjects would be recruited in future research to definitively evaluate these findings. Second, we chose ALFF and DC as the indices to detect the fluctuations in regional spontaneous activity strength. According to these two measures, the brain of amputation patients showed large-scale changes over 12 months. To clarify the underlying neural mechanisms related to amputation-related brain changes, future studies should examine alterations at the brain network level. Finally, clinical assessment with validated neuropsychological instruments should be performed in future studies, which could uncover a more interesting relationship between amputation and the changed BOLD signals.

\section{Conclusion}

Our findings show that local and extensive temporal and spatial changes occur in intrinsic cortical activity and functional connectivity in the sensorimotor and cognitive-related brain regions after limb amputation. With future confirmatory research, this plasticity may imply that dysfunction occurs not only in sensory and motor regions but also in areas responsible for sensorimotor integration and motor planning. Regardless, these results and others' on how ALFF and DC are plastic after traumatic limb amputation highlight how analyzing intrinsic brain activity in the absence of any task-related sensory or cognitive stimulus (rs-fMRI) may be a fruitful approach for analyzing plasticity after "natural deafferentation" that occurs in Alzheimer's disease or Parkinson's disease.

\section{Data Availability}

The present study includes 8 healthy volunteers of 5 men and 3 women (mean age: 37.9 years old) and 16 patients with amputation (11 men and 5 women; mean age: 41.2 years old). All procedures used in this study were approved by the Committee for Medical Ethics of Shanghai Jiao Tong University Affiliated Sixth People's Hospital. Written informed consent was obtained from all study participants.

\section{Disclosure}

This article has been accepted for publication in Preprint by Research Square. Bingbo Bao is the first author. Lei Duan is the co-first author. Haifeng Wei, Pengbo Luo, Hongyi Zhu, Tao Gao, Xiaoer Wei, Jing Li, Yuehua Li, Yimin Chai, and Changqing Zhang are the joint co-authors.

\section{Conflicts of Interest}

All authors declare no competing interests.

\section{Authors' Contributions}

Bingbo Bao and Lei Duan contributed equally to this work. Xianyou Zheng was responsible for the study design and manuscript writing. Bingbo Bao and Lei Duan were responsible for the data collection and analysis. Bingbo Bao was responsible for the revision of the manuscript. All the authors critically reviewed the content of the manuscript. All authors read and approved the final manuscript.

\section{Acknowledgments}

This study was supported by the Shanghai Municipal Education Commission-Gaofeng Clinical Medicine Grant (grant number 20161429) and the National Natural Science Foundation of China (grant number 81371965). 


\section{References}

[1] S. M. Grüsser, C. Winter, W. Mühlnickel et al., "The relationship of perceptual phenomena and cortical reorganization in upper extremity amputees," Neuroscience, vol. 102, no. 2, pp. 263-272, 2001.

[2] A. Karl, N. Birbaumer, W. Lutzenberger, L. G. Cohen, and H. Flor, "Reorganization of motor and somatosensory cortex in upper extremity amputees with phantom limb pain," The Journal of Neuroscience, vol. 21, no. 10, pp. 3609-3618, 2001.

[3] L. G. Cohen, B. J. Roth, E. M. Wassermann et al., "Magnetic stimulation of the human cerebral cortex, an indicator of reorganization in motor pathways in certain pathological conditions," Journal of Clinical Neurophysiology, vol. 8, no. 1, pp. 56-65, 1991.

[4] H. Flor, T. Elbert, S. Knecht et al., "Phantom-limb pain as a perceptual correlate of cortical reorganization following arm amputation," Nature, vol. 375, no. 6531, pp. 482-484, 1995.

[5] P. Giraux, A. Sirigu, F. Schneider, and J. M. Dubernard, "Cortical reorganization in motor cortex after graft of both hands," Nature Neuroscience, vol. 4, no. 7, pp. 691-692, 2001.

[6] J. I. Romero-Romo, C. C. C. Bauer, E. H. Pasaye, R. A. Gutiérrez, R. Favila, and F. A. Barrios, "Abnormal functioning of the thalamocortical system underlies the conscious awareness of the phantom limb phenomenon," The Neuroradiology Journal, vol. 23, no. 6, pp. 671-679, 2010.

[7] E. H. Pasaye, R. A. Gutiérrez, S. Alcauter et al., "Event-related functional magnetic resonance images during the perception of phantom limb. A brushing task," The Neuroradiology Journal, vol. 23, no. 6, pp. 665-670, 2010.

[8] V. S. Ramachandran, M. Stewart, and D. C. Rogers-Ramachandran, "Perceptual correlates of massive cortical reorganization," Neuroreport, vol. 3, no. 7, pp. 583-586, 1992.

[9] V. S. Ramachandran and W. Hirstein, "The perception of phantom limbs. The D. O. Hebb lecture," Brain, vol. 121, no. 9, pp. 1603-1630, 1998.

[10] H. Flor, L. Nikolajsen, and T. Staehelin Jensen, "Phantom limb pain: a case of maladaptive CNS plasticity?," Nature Reviews Neuroscience, vol. 7, no. 11, pp. 873-881, 2006.

[11] T. R. Makin, N. Filippini, E. P. Duff, D. Henderson Slater, I. Tracey, and H. Johansen-Berg, "Network-level reorganisation of functional connectivity following arm amputation," NeuroImage, vol. 114, pp. 217-225, 2015.

[12] T. R. Makin, J. Scholz, N. Filippini, D. Henderson Slater, I. Tracey, and H. Johansen-Berg, "Phantom pain is associated with preserved structure and function in the former hand area," Nature Communications, vol. 4, no. 1, article 1570, 2013.

[13] T. R. Makin, A. O. Cramer, J. Scholz et al., "Deprivationrelated and use-dependent plasticity go hand in hand," eLife, vol. 2, article e01273, 2013.

[14] C. D. Vargas, A. Aballéa, E. C. Rodrigues et al., "Re-emergence of hand-muscle representations in human motor cortex after hand allograft," Proceedings of the National Academy of Sciences of the United States of America, vol. 106, no. 17, pp. 7197-7202, 2009.

[15] E. L. Simões, I. Bramati, E. Rodrigues et al., "Functional expansion of sensorimotor representation and structural reorganization of callosal connections in lower limb amputees," The Journal of Neuroscience, vol. 32, no. 9, pp. 3211-3220, 2012.

[16] B. Biswal, F. Zerrin Yetkin, V. M. Haughton, and J. S. Hyde, "Functional connectivity in the motor cortex of resting human brain using echo-planar MRI," Magnetic Resonance in Medicine, vol. 34, no. 4, pp. 537-541, 1995.

[17] R. van Veen, V. Gurvits, R. V. Kogan et al., “An application of generalized matrix learning vector quantization in neuroimaging," Computer Methods and Programs in Biomedicine, vol. 197, p. 105708, 2020.

[18] S. Ogawa, R. S. Menon, D. W. Tank et al., "Functional brain mapping by blood oxygenation level-dependent contrast magnetic resonance imaging. A comparison of signal characteristics with a biophysical model," Biophysical Journal, vol. 64, no. 3, pp. 803-812, 1993.

[19] S. Ogawa, T. M. Lee, A. R. Kay, and D. W. Tank, "Brain magnetic resonance imaging with contrast dependent on blood oxygenation," Proceedings of the National Academy of Sciences of the United States of America, vol. 87, no. 24, pp. 9868-9872, 1990.

[20] T. T. Liu, "Neurovascular factors in resting-state functional MRI," NeuroImage, vol. 80, pp. 339-348, 2013.

[21] Y. Chao-Gan and Z. Yu-Feng, "DPARSF: a MATLAB toolbox for "pipeline" data analysis of resting-state fMRI," Frontiers in Systems Neuroscience, vol. 4, p. 13, 2010.

[22] X. W. Song, Z. Y. Dong, X. Y. Long et al., "REST: a toolkit for resting-state functional magnetic resonance imaging data processing," PLoS One, vol. 6, no. 9, article e25031, 2011.

[23] X. N. Zuo, A. Di Martino, C. Kelly et al., "The oscillating brain: complex and reliable," NeuroImage, vol. 49, no. 2, pp. 1432 $1445,2010$.

[24] Z. Yu-Feng, H. Yong, Z. Chao-Zhe et al., "Altered baseline brain activity in children with ADHD revealed by resting- state functional MRI," Brain \& Development, vol. 29, no. 2, pp. 8391, 2007.

[25] Y. Zang, T. Jiang, Y. Lu, Y. He, and L. Tian, "Regional homogeneity approach to fMRI data analysis," NeuroImage, vol. 22, no. 1, pp. 394-400, 2004.

[26] X. N. Zuo, R. Ehmke, M. Mennes et al., "Network centrality in the human functional connectome," Cerebral Cortex, vol. 22, no. 8, pp. 1862-1875, 2012.

[27] M. W. Cole, S. Pathak, and W. Schneider, "Identifying the brain's most globally connected regions," NeuroImage, vol. 49, no. 4, pp. 3132-3148, 2010.

[28] R. L. Buckner, J. Sepulcre, T. Talukdar et al., "Cortical hubs revealed by intrinsic functional connectivity: mapping, assessment of stability, and relation to Alzheimer's disease," The Journal of Neuroscience, vol. 29, no. 6, pp. 1860-1873, 2009.

[29] S. Li, X. Ma, R. Huang et al., "Abnormal degree centrality in neurologically asymptomatic patients with end- stage renal disease: a resting-state fMRI study," Clinical Neurophysiology, vol. 127, no. 1, pp. 602-609, 2016.

[30] M. D. Fox, D. Zhang, A. Z. Snyder, and M. E. Raichle, "The global signal and observed anticorrelated resting state brain networks," Journal of Neurophysiology, vol. 101, no. 6, pp. 3270-3283, 2009.

[31] K. Murphy, R. M. Birn, D. A. Handwerker, T. B. Jones, and P. A. Bandettini, "The impact of global signal regression on resting state correlations: are anti-correlated networks introduced?," NeuroImage, vol. 44, no. 3, pp. 893-905, 2009.

[32] X. N. Zuo, C. Kelly, A. Di Martino et al., "Growing together and growing apart: regional and sex differences in the lifespan developmental trajectories of functional homotopy," The Journal of Neuroscience, vol. 30, no. 45, pp. 1503415043, 2010. 
[33] M. M. Merzenich, R. J. Nelson, M. P. Stryker, M. S. Cynader, A. Schoppmann, and J. M. Zook, "Somatosensory cortical map changes following digit amputation in adult monkeys," The Journal of Comparative Neurology, vol. 224, no. 4, pp. 591-605, 1984.

[34] J. P. Donoghue and J. N. Sanes, "Peripheral nerve injury in developing rats reorganizes representation pattern in motor cortex," Proceedings of the National Academy of Sciences of the United States of America, vol. 84, no. 4, pp. 1123-1126, 1987.

[35] C. W. H. Wu and J. H. Kaas, "Reorganization in primary motor cortex of primates with long-standing therapeutic amputations," The Journal of Neuroscience, vol. 19, no. 17, pp. 7679-7697, 1999.

[36] L. G. Cohen, S. Bandinelli, T. W. Findley, and M. Hallett, "Motor reorganization after upper limb amputation in man," Brain, vol. 114, no. 1, pp. 615-627, 1991.

[37] S. Roricht, B. U. Meyer, L. Niehaus, and S. A. Brandt, "Longterm reorganization of motor cortex outputs after arm amputation," Neurology, vol. 53, no. 1, pp. 106-111, 1999.

[38] M. Lotze, H. Flor, W. Grodd, W. Larbig, and N. Birbaumer, "Phantom movements and pain an fMRI study in upper limb amputees," Brain, vol. 124, no. 11, pp. 2268-2277, 2001.

[39] T. Elbert, H. Flor, N. Birbaumer et al., "Extensive reorganization of the somatosensory cortex in adult humans after nervous system injury," NeuroReport, vol. 5, no. 18, pp. 2593-2597, 1994.

[40] C. Dettmers, T. Adler, R. Rzanny et al., "Increased excitability in the primary motor cortex and supplementary motor area in patients with phantom limb pain after upper limb amputation," Neuroscience Letters, vol. 307, no. 2, pp. 109-112, 2001.

[41] J. A. Turner, J. S. Lee, O. Martinez, A. L. Medlin, S. L. Schandler, and M. J. Cohen, "Somatotopy of the motor cortex after longterm spinal cord injury or amputation," IEEE Transactions on Neural Systems and Rehabilitation Engineering, vol. 9, no. 2, pp. 154-160, 2001.

[42] F. E. Roux, J. A. Lotterie, E. Cassol, Y. Lazorthes, J. C. Sol, and I. Berry, "Cortical areas involved in virtual movement of phantom limbs: comparison with normal subjects," Neurosurgery, vol. 53, no. 6, pp. 1342-1353, 2003.

[43] T. Aflalo, S. Kellis, C. Klaes et al., "Neurophysiology. Decoding motor imagery from the posterior parietal cortex of a tetraplegic human," Science, vol. 348, no. 6237, pp. 906-910, 2015.

[44] S. N. Flesher, J. L. Collinger, S. T. Foldes et al., "Intracortical microstimulation of human somatosensory cortex," Science Translational Medicine, vol. 8, no. 361, article 361ra141, 2016.

[45] S. Kikkert, J. Kolasinski, S. Jbabdi et al., "Revealing the neural fingerprints of a missing hand," eLife, vol. 5, article e15292, 2016. 